\title{
Disseminating Evidence-Based Psychological Treatments for Eating Disorders
}

\author{
Zafra Cooper • Suzanne Bailey-Straebler
}

Published online: 7 February 2015

(C) The Author(s) 2015. This article is published with open access at Springerlink.com

\begin{abstract}
The research-practice gap is of concern in the treatment of eating disorders. Despite the existence of empirically supported treatments, few receive them. The barriers to wider dissemination and implementation of evidence-based treatment include clinician attitudes towards such treatments and the lack of sufficient numbers of suitably trained therapists to provide treatment. In this review we discuss these barriers in the context of the wider issue of the dissemination and implementation of psychological treatments and review the research with regard to the treatment of eating disorders. Particular emphasis is placed on examining recent efforts to expand the availability and reach of treatments by making treatment delivery and training more scalable. We highlight promising developments and areas where further research is needed.
\end{abstract}

Keywords Eating disorders $\cdot$ Empirically supported treatment $\cdot$ Dissemination $\cdot$ Implementation $\cdot$ Scalable training

\section{Introduction}

The research-practice gap has been well documented in recent years. Treatment researchers and developers as well as those

This article is part of the Topical Collection on Eating Disorders

Z. Cooper $(\bowtie) \cdot$ S. Bailey-Straebler

Department of Psychiatry, Warneford Hospital, Oxford University, Oxford OX3 7JX, UK

e-mail: zafra.cooper@psych.ox.ac.uk

S. Bailey-Straebler

e-mail: suzanne.straebler@psych.ox.ac.uk involved in formulating public policy have highlighted the problem in medicine generally and in the area of mental health in particular [1-5]. Despite the considerable progress that has been made in the development and empirical evaluation of psychological treatments, there is agreement that this gap needs to close if such treatments are to achieve their promise for public health benefit. The need to focus on both dissemination, defined here as the process of ensuring the adoption of treatments, and implementation, the process of translating these treatments into routine and persisting clinical practice, is further supported by evidence suggesting that even when patients do receive empirically supported treatments ${ }^{1}$, they are not always well delivered [3]. The research-practice gap is of particular concern in the treatment of eating disorders $[6 \cdot \bullet]$.

\section{Eating Disorders- the Need for Dissemination and Implementation}

The eating disorders, anorexia nervosa, bulimia nervosa, binge eating disorder, and their variants are serious disorders that are accompanied by significant impairment in physical and psychological functioning as well as in quality of life [7]. Over the past two decades, significant progress has been made in developing treatments for these disorders and evidence for their efficacy has been documented in both narrative and systematic reviews. In particular, cognitive behavioural therapy (CBT), a guided self-help form (GSH) of CBT, enhanced cognitive behavioural treatment (CBT-E) and interpersonal psychotherapy (IPT) are recommended for the treatment of bulimia nervosa, binge eating disorder and to a lesser extent the atypical eating disorders [8-13], with further support for

\footnotetext{
${ }^{1}$ It should be noted that the terms evidence-based, evidence supported and empirically supported are all used in the literature. They are often used interchangeably, and we have followed this practice.
} 
CBT-E from a recently published study [14]. Treatment for adults with anorexia nervosa is less well supported [11-13, 15, 16] with a number of approaches including CBT-E and IPT showing some promise and newer treatments such as the Maudsley model for treatment of adults with anorexia nervosa (MANTRA) and cognitive remediation therapy (CRT) under further investigation $[12,13]$. As yet, one specialist treatment has not emerged as clearly superior to the others, with a recently published study [17] being broadly consistent with this conclusion, although the version of CBT-E used differs from the published manual [18]. For adolescents with anorexia nervosa, family-based treatment (FBT) has received the most support [13, 19, 20] with preliminary support for CBT-E [13].

Yet despite the existence of these empirically supported treatments for eating disorders, they are not reaching those who need them. In part, this is due to individuals not seeking treatment or eating problems not being correctly detected in primary care $[21,22]$. However, even when treatment is sought and offered, many do not receive empirically supported interventions [22-28]. In sum, the disconnect between research and practice is as much a problem in the eating disorders as it is for psychological treatments in general.

\section{The Nature of the Problem}

The barriers to the dissemination and implementation of empirically supported treatments for eating disorders are not unique, with similar problems occurring in the case of most psychological treatments. Broadly, the barriers divide into those concerning attitudes and beliefs of practitioners and organisations about adopting and implementing new evidencebased treatments $[5,29,30]$ and issues relating to the availability of and access to treatment [4, 31•]. Overcoming barriers of availability raises obstacles in the form of gaps in our knowledge about how treatments are best delivered and how training should be provided. We discuss these two main kinds of barrier in relation to treatments for eating disorders, concentrating particularly on recent efforts to expand the availability and reach of treatments by making treatment delivery and training more scalable. While the work on prevention of eating disorders clearly has an important role to play in the project of reducing the burden of disorder, it is not within the scope of the current review.

\section{Attitudes Towards the Use of Empirically Supported Treatments for Eating Disorders}

While clinician and organisational attitudes that may be a barrier to the adoption of evidence-based psychological treatments in general have been fairly extensively discussed as noted above, the discussion of this issue in the field of eating disorder treatment is relatively recent. Studies have examined eating disorder clinicians' attitudes and concerns about the use of empirically supported treatments and manual use in particular, and the attributes of those clinicians who are more likely to use such treatments.

Concerns About the Use of Empirically Supported Treatments and Manuals

A number of recent studies of clinicians treating eating disorders have explicitly reported their reservations and concerns about the use of evidence-supported treatment. Two internationally based large surveys of eating disorder clinicians [23, 32] reported that concerns about the generalizability of research findings led clinicians to modify treatment in clinical practice, with only very small numbers reporting that they adhered closely to a manual. Consistent with these observations are reports that clinicians claim to use CBT but do not use it as their primary approach [33] or that they use an eclectic approach combining elements of evidence-based treatment with approaches that are not empirically supported [25]. Clinicians generally expressed the view that manual-based treatments were too rigid and constraining to be a good fit for their patients while lack of sufficient training and inconsistency with their own theoretical orientation were lesser concerns.

Two more recent studies concentrated on clinicians in two single countries. A survey of clinicians working in publicly funded specialist eating disorder clinics in the United Kingdom (UK) found that negative attitudes towards treatment manuals and the potential outcomes they might achieve were associated with three beliefs: that manuals did not stress the therapeutic alliance; that they did not contain clinical case examples, and that they were imposed by third-party payers [34]. An interview-based study of community clinicians treating eating disorders across one Canadian province found that CBT was not used because the majority of therapists did not regard it as consistent with their theoretical orientation or their personal clinical style. Many also reported that their clinical experience suggested that CBT was not effective, while a smaller proportion believed it was inflexible [26]. In contrast, the main reason therapists gave for not using IPT was lack of training.

Qualitative studies provide an opportunity to explore and understand clinicians' attitudes in greater depth. One such study [27, 28] investigated the uptake of FBT amongst clinicians treating children and adolescents with anorexia nervosa and found that clinicians expressed concerns about implementing some specific aspects of the intervention (weighing, nutritional advice and family meals) and many reported the commonly held objection that "one size does not fit all". They also cited organisational factors stressing that adoption of FBT would be facilitated by support from clinical managers. Another recent qualitative study [35] found that clinicians who had attended a training workshop on CBT-E 
were broadly positive about empirically supported treatment but did not think that it was appropriate to implement the treatment in its entirety in their routine practice.

Characteristics Associated With the Use of Empirically Supported Treatments and Manuals

The literature on the characteristics, demography and psychology of those that use evidence-based treatment and manuals is fairly limited. Data from a large international survey of eating disorder clinicians and researchers suggests that manual use in the treatment of bulimia nervosa is more likely if clinicians are: younger; psychologists; involved in research and treating adult patients [36]. A study of clinicians who reported routinely offering CBT for eating disorders in the UK produced broadly consistent findings in that older, more experienced and more anxious clinicians reported using fewer of the core elements of evidence-supported CBT [24].

Two further studies report on the relationship between clinician's emotional characteristics and their attitudes towards the use of evidence-supported manuals or particular elements of evidence-based treatment. Perhaps unsurprisingly, those clinicians with negative attitudes towards manuals had higher scores on depressed mood [34] and concern about implementing certain key features of evidence-supported treatment amongst those offering CBT was related to clinicians' anxiety levels, with older more experienced clinicians generally being less concerned about their use [37].

\section{Implications of Clinicians' Understanding of the Empirical Evidence}

Even when clinicians are broadly supportive of an evidencebased approach, they may have a variety of different interpretations about the nature of the existing evidence and about how it should be used in treating their patients. A belief that findings from research studies may have limited applicability for their patients often leads them to rely exclusively on their own clinical judgement rather than research findings and to combine eclectically both empirically supported and unsupported treatments with little regard for the potential disadvantages of this approach. Furthermore, a belief in the importance of the therapeutic alliance above the content of the protocol and particular concern about certain interventions and the effects they may have on their patients may also contribute to widespread lack of adherence to evidence-supported practice.

Some of these concerns may be addressed by highlighting research studies that have had relatively few exclusion criteria for trial entry (e.g., [38]). More importantly, recent effectiveness studies in routine clinical settings have reported results largely consistent with research trial findings [39-42], albeit it with higher drop-out rates. Nevertheless, more information is needed from controlled trials on the use of treatments in routine clinical settings. Relatively little is known about the key mechanisms of action of treatments for eating disorders. Such knowledge would enable us to preserve effective treatment elements and eliminate redundant ones when adapting or simplifying treatments for more widespread routine use. Ongoing work on mediators of change [43] will be helpful in this regard.

A solution often offered for overcoming attitudinal barriers has been further training and ongoing supervision. There has been relatively little research on how to train eating disorder therapists (see further discussion below). We suggest that training should focus both on acquiring knowledge about treatments and their implementation and on a better understanding of the nature of the evidence supporting these treatments and how to interpret and use it.

\section{Expanding the Availability of Empirically Supported Treatment for Eating Disorders}

In common with many other forms of psychological treatment, the evidence supporting treatments for eating disorders is derived from studies in which treatment is delivered individually or in small groups by highly trained mental health professionals meeting with patients in a face-to-face setting. Furthermore, research findings generally relate to a specific eating disorder diagnostic category. This is consistent with what is still the dominant model of treatment delivery. However, this model of delivery has been criticised because it is unlikely to be sufficiently scalable to meet the needs of all those who need treatment [44••]. At the same time, it has been noted that such treatments are inherently difficult to scale up $[45 \cdot \bullet]$. In the eating disorders, two major problems are highlighted: even in resource-rich countries, there are major geographic and demographic inequalities in the availability of treatments [46], and the problem is even more acute in resource-poor countries; and, more fundamentally, there are not sufficient numbers of therapists appropriately trained to deliver these treatments [31•] nor is training readily available. A number of interesting initiatives have been proposed to address these two problems, only some of which have been tried and tested within the field of eating disorders.

A recent commentary detailing a "road map" for closing the research-practice gap suggests that initiatives to increase the availability of empirically supported treatments may be considered under two broad headings, those that remain essentially therapist led and those that are programme led [45••]. In the former case, the treatment content is delivered by a therapist although content, delivery medium or method of therapist training (which itself may be either trainer led or programme led, as discussed below) may be modified to make treatment more widely available. In the latter case, treatment 
content is conveyed directly to the individual by a variety of means (including books, CD-ROMs, downloadable and online resources) and may or may not be accompanied by the help of a guide. These alternatives can be viewed as existing along a continuum of increasing scalability (see Fig. 1).

\section{Therapist-Led Treatment for Eating Disorders}

\section{Changing the Mode of Treatment Delivery for Difficult} to Reach Populations

Simply changing the mode of delivery of therapist-led interventions has the potential to reach populations in areas where there are few therapists as well as patient groups who, for a variety of reasons, would not or could not attend face-to-face sessions. Two such approaches are of note. A study comparing 20-week manual-based face-to-face CBT for the treatment of bulimia nervosa $(\mathrm{BN})$ with the same treatment delivered via telemedicine found the latter treatment to be cheaper with no differences in treatment outcome [46, 47]. An ongoing noninferiority study is investigating 20-week group CBT, delivered either in face-to-face form or as a moderated chat group delivered over the internet, in terms of the efficacy, acceptability, attrition rate and cost effectiveness of the two treatments [48]. While these approaches clearly have advantages in making treatment more available, they still rely on trained eating disorder therapists who are in short supply.

\section{Training More Therapists}

Of course, the most straightforward solution to a shortage of appropriately trained therapists is to train more of them. This has been the solution adopted by a number of large-scale dissemination and implementation projects such as the Improving Access to Psychological Treatments (IAPT) initiative across England [49] and the programmes adopted by the Veterans Health Administration [50]. There is no such similar project dedicated to dissemination and implementation of eating disorder treatments, and besides, projects such as these rely on the availability of large-scale resources to provide training and supervision.

In general, the topic of therapist training has been relatively neglected in the research literature and similarly the related issue of the measurement of the outcome of training has also been largely overlooked [51]. This applies also in the case of eating disorders. Generally, it is agreed that at least three components of training are necessary: attending a workshop led by an expert in the treatment; detailed study of a treatment manual and, usually thought most important, practising the treatment with ongoing expert supervision [52, 53]. In treatment studies, there may also be feedback on therapy quality from experts listening to recordings of sessions. While indirect evidence from patient outcome suggests that this is an effective form of training, this method is time consuming and depends on the availability of scarce and costly experts to provide workshops and supervision.

For sufficient numbers of eating disorder therapists to be trained, it is necessary to develop and evaluate models of training that are potentially more scalable. Possible ways of achieving this are to investigate training a wider range of therapists, to explore whether therapists may be trained in skills that are more widely generalizable and, perhaps most importantly, whether it is feasible for training to be programme led rather than trainer led (see Fig. 1). It is worth noting that the empirical evaluation of different methods of training (in a move towards evidencesupported training) raises the question of measuring the outcome of training [54]. A detailed discussion of the assessment of the outcome of training is beyond the scope of the present review.

\section{Who Should Be Trained?}

When considering who should be trained, two approaches hold promise for increasing the availability of trained therapists. The first, the train-the-trainer model makes use of an expert trainer to pass on skills to a range of less expert trainers who in turn can train larger numbers of therapists [53]. The

Fig. 1 The relationship between scalable treatment and scalable training

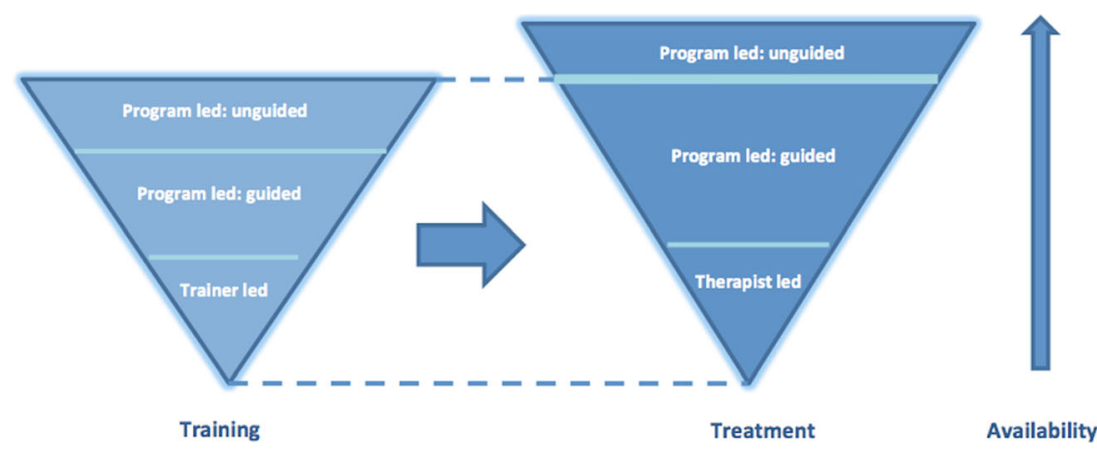


second, more radical task shifting approach, aims to train a wider range of potential therapists to take on tasks usually performed only by those with specialist mental health qualifications $[44 \bullet \bullet, 55]$. By devolving specialist roles to less specialist or non-specialist therapists trained in particular interventions, the pool of potential trainees is increased, and almost certainly, the overall cost of training is decreased.

These approaches have barely been investigated in the field of eating disorder treatment. A proof of concept study investigated the outcome of guided self-help for those with binge eating problems in an open clinical trial where the training and supervision of the graduate student therapists was provided by a master's level clinical psychology graduate who had been trained by an expert [56]. Although this train-the-trainer model appeared to be a feasible and acceptable strategy and results were consistent with those reported in the literature for selfhelp treatment, it should be noted that this study investigated a programme-led approach (guided self-help) rather than a therapist-led approach. A similar approach has not been investigated for a therapist-led treatment. Of concern is the question of whether train-the-trainer approaches can meet the scale of the problem [57].

To our knowledge, the task shifting approach has not been systematically investigated in training eating disorder therapists. The obvious objection to this approach is that it risks compromising the quality of care provided. Reports from the area of general medicine and early indications from the use of this strategy in mental health interventions in resource-poor environments suggest that it may not [58]. As such, it is a method worth investigating in training eating disorder therapists.

\section{What Should Therapists Be Trained to Do?}

As noted earlier, the dominant model of treatment delivery requires that therapists learn a number of different evidence supported treatments to provide the appropriate intervention to fit particular diagnostic categories. It has been argued that the need to train in a variety of single-disorder approaches may be unrealistic [59]. Transdiagnostic treatments (e.g., $[60,61])$ provide the possibility of learning one empirically supported treatment, which may be applied flexibly to a range of psychopathology rather than to a particular diagnostic category. Similarly, modular treatments and principle- or component-based approaches may offer similar advantages $[62,63]$, although these have not been systematically investigated in eating disorders.

As noted earlier, CBT-E, a treatment designed to be transdiagnostic in scope, has received support in several RCTs [14, 38, (Fairburn, Bailey-Straebler, Basden, Doll, Jones, Murphy, O'Connor and Cooper, submitted)] although support for its use with patients who are significantly low in weight is still preliminary [64].

\section{How Should Therapists Be Trained?}

Programme-led rather than trainer-led methods of training have the potential to reach many more trainees. As noted, treatment manuals tend not to be used by large number of therapists. The availability of a range of modern methods of communication has created the possibility of programme-led approaches that might have several advantages over bookbased treatment guides. One such approach potentially capable of training large numbers of therapists is web-centred training $[51,65,66]$. Details of treatment, guidance about how it should be implemented and illustrations of key procedures can be provided in interactive form online or in the form of downloadable material. Advantages of web-centred training include trainees being able to work through the training programme at their own pace, return to key material should they wish and potentially view many acted illustrations of clinical interventions. The training could possibly be undertaken with the help of guides who are not expert clinicians and who do not provide clinical supervision. Rather, their role would be to help trainees use the training programme effectively by motivating them to complete the training and implement treatment with their patients. Alternatively, training could be undertaken independently without a guide and it is possible that at least a proportion of trainees could benefit from this entirely scalable form of training.

Web-centred training has not yet been evaluated in the training of eating disorder therapists. We have recently completed a large pilot study in which one hundred therapists from a range of professional groups received guided web-based training in CBT-E using a newly developed training website. Preliminary results suggest that the method of training is feasible and acceptable to trainees, with very few trainees failing to complete training. Our group is currently comparing guided web-centred training with unguided independent training in an ongoing randomised controlled trial.

\section{Programme-Led Treatment for Eating Disorders}

Even if training becomes much more available than at present, programme-led self-help approaches have the advantage of being able to reach a wider range of those who need treatment in a cost-effective way, including those who might not seek it through the usual clinical routes [45••, 67]. Additional benefits include the individual being able to pursue treatment at their own pace and a sense of empowerment [68]. Self-help interventions that involve an individual independently following a treatment programme, presented in either book or electronic form without any further support, have the most potential for wide or even global reach. Guided self-help involving varying degrees of input from a mental health professional or 
guide also has such potential, limited of course by the nature and amount of guidance provided, the qualifications of the guide and the amount of training and supervision received.

\section{Guided Self-Help}

There is good evidence to support self-help versions of a number of empirically supported psychological treatments $[69,70]$. Most recent work has focused on guided self-help rather than unguided or pure self-help, although available research findings do not conclusively favour guided self-help over unguided self-help $[31 \bullet, 71]$. A detailed recent review of the use of self-help to treat eating disorders found that there was good evidence for the use of guided self-help based on CBT principles for the treatment of binge eating disorder, that the evidence was less good but promising for the treatment of bulimia nervosa and that it was contraindicated in the treatment of anorexia nervosa [31•]. There is much less consistent evidence that it is as effective as therapist-led treatment. However, from the point of view of reducing the unmet need for effective treatments for eating disorders, a smaller effect size that can be widely and reliably achieved may be of vital importance [4].

One question that needs further consideration is the extent to which programme-led guided self-help approaches for the treatment of eating disorders are indeed scalable. While guided self-help is generally delivered by a wider set of providers than those that offer treatment, self-help guides tend to be health professionals (e.g., nurses) or trainee mental health professionals (graduate clinical psychology trainees, psychiatry residents, etc.) [31•]. With a few exceptions (see below), it has frequently been offered in face-to-face settings or by one-to-one telephone contacts in the context of university based research studies or research clinics [72]. Furthermore, the training and supervision received by guides varies greatly and is often not reported [73]. It is instructive to note that that the optimal training proposed for implementing guided selfhelp involves training and ongoing supervision or patient monitoring. It is judged optimal because it is associated with the best outcome. Of course, methods discussed earlier such as task shifting and web-based training may contribute to making guided self-help more scalable than it is at present (see Fig. 1).

\section{Unguided Self-Help}

Self-help treatments may be more scalable if delivered primarily through unguided electronic or book-based approaches. At present, internet access is not universal and in certain areas and, for certain populations, self-help books may still be the most easily and widely available option. However, unguided treatment also raises a range of ethical and legal issues, which will need careful consideration [74].
Three recent systematic reviews have been published investigating self-help treatments $[75,76,77 \cdot]$ delivered primarily by electronic means. While two of these reviews concluded that the internet was a promising method of treating eating disorders and even a good alternative to individual treatment [76], the third review using National Institute for Health and Care Excellence (NICE) methodology was much more cautious, concluding that the status of e-therapy was still uncertain for $\mathrm{BN}$ and binge eating disorder (BED) and unknown for anorexia nervosa (AN) [77॰]. The use of mobile devices (mobile apps) either as an adjunct to treatment or as a means to deliver treatment has yet to be systematically explored [77•, 78•]. As in previous studies of self-help interventions, studies varied to the extent that they provided added support from a health professional or other guide.

Finally, two recent studies investigated unguided or pure self-help using a self-help book $[72,79]$ in a diverse group of patients with BED in primary care. In neither study was unguided self-help better than the control conditions.

Newer more scalable e-therapy versions of self-help are in the early stages of development and might benefit from using the full range of modern communication resources rather than simply transferring book-based material to an online format. Given the potential of various forms of unguided self-help for widespread use, further research attention is merited. Developing effective self-help interventions that can be used with little or no expert support and determining for whom they are likely to be helpful is an urgent priority. In particular, it is important to specify the amount and nature of any additional support participants are given when following these programmes including specifying details of the training and ongoing supervision provided for guides.

\section{Conclusion}

The well-documented unmet need for eating disorder treatment together with growing evidence that patients do not receive empirically supported treatments has focused research attention on ensuring that such treatments are more widely available and used. Over recent years, relatively more attention has been directed towards developing and evaluating a range of programme-led self-help approaches rather than on investigating ways of increasing the availability of therapistled approaches and training. We suggest that both are needed.

While self-help treatment holds the greatest promise for reaching those in need of treatment, it has not been shown to be suitable for the full range of eating disorders. New programme-led treatment interventions need to be subject to the same rigorous evaluation as demanded for other psychological treatments, as well as a systematic examination of their costs and public health impact. It is important to note that when evaluating scalable treatment, or indeed training 
interventions, non-inferiority designs [78•] are likely to be important. For programme-led treatment interventions, more use of "direct-to-user" recruitment [77•] is desirable if such interventions are to be shown to be truly scalable. Finally, determining for whom programme-led approaches are suitable is a priority.

Given that therapist-led treatment will continue to be required, there is also an urgent need for evidence-based training. In particular, new programme-based training methods need to be developed and rigorously evaluated. Training needs to address the problem of training more therapists by exploring further the use of train-the-trainer models, task shifting and transdiagnostic protocols where possible. There is also a need to further understand therapists' concerns about using empirically supported treatments and address these in training. Programme-led approaches such as web-centred training hold promise for making training more widely available, but it has to be established that they do not compromise the quality of training therapists receive. The outcome of such training needs careful evaluation together with a more detailed study of therapist competence and its relation to patient outcome. Finally, further research on developing measures of therapist competence is required.

Acknowledgments The authors are supported by a Strategic Award from the Wellcome Trust, London (094585).

\section{Compliance with Ethics Guidelines}

Conflict of Interest Both authors are supported by the Wellcome Trust who had no further role in the preparation of the manuscript

Human and Animal Rights and Informed Consent This article does not contain any studies with human or animal subjects performed by any of the authors.

Open Access This article is distributed under the terms of the Creative Commons Attribution License which permits any use, distribution, and reproduction in any medium, provided the original author(s) and the source are credited.

\section{References}

Papers of particular interest, published recently, have been highlighted as:

- Of importance

•- Of major importance

1. Institute of Medicine. Crossing the quality chasm: a new health system for the 21st century. Committee on Quality of Health Care in America. Washington DC: National Academies Press; 2001.

2. Insel TR. Translating scientific opportunity into public health impact: a strategic plan for research on mental illness. Archives Gen Psychiatr. 2009;66(2):128-33.
3. Shafran R et al. Mind the gap: improving the dissemination of CBT. Behav Res Ther. 2009;47(11):902-9.

4. Kazdin AE, Blase SL. Rebooting psychotherapy research and practice to reduce the burden of mental illness. Perspect Psychol Sci. 2011;6(1):21-37.

5. McHugh RK, Barlow DH. The reach of evidence-based psychological interventions. In: McHugh RK, Barlow DH, editors. Dissemination and implementation of evidence-based psychological interventions. New York: Oxford University Press; 2012. p. 315.

$6 . \bullet$ Lilienfeld SO. The research-practice gap: bridging the schism between eating disorder researchers and practitioners. Int J Eat Disord. 2013;46(5):386-94. An interesting discussion of the researchpractice gap in the treatment of eating disorders highlighting, in particular, the role of clinician attitudes.

7. Klump KL et al. Academy for eating disorders position paper: eating disorders are serious mental illnesses. Int J Eat Disord. 2009;42(2):97-103.

8. National Institute for Health and Care Excellence. National clinical practice guideline: eating disorders - core interventions in the treatment and management of anorexia nervosa, bulimia nervosa and related eating disorders. London; 2004.

9. Shapiro JR et al. Bulimia nervosa treatment: a systematic review of randomized controlled trials. Int J Eat Disord. 2007;40(4):321-36.

10. Brownley KA et al. Binge eating disorder treatment: a systematic review of randomized controlled trials. Int J Eat Disord. 2007;40(4): $337-48$.

11. Wilson GT, Grilo CM, Vitousek KM. Psychological treatment of eating disorders. Am Psychol. 2007;62(3):199-216.

12. Hay P. A systematic review of evidence for psychological treatments in eating disorders: 2005-2012. Int J Eat Disord. 2013;46(5):462-9.

13. Kass AE, Kolko RP, Wilfley DE. Psychological treatments for eating disorders. Curr Opin Psychiatr. 2013;26(6):549-55.

14. Poulsen $\mathrm{S}$ et al. A randomized controlled trial of psychoanalytic psychotherapy or cognitive-behavioral therapy for bulimia nervosa. Am J Psychiatry. 2014;107(1):109-16.

15. Bulik CM et al. Anorexia nervosa treatment: a systematic review of randomized controlled trials. Int J Eat Disord. 2007;40(4):310-20.

16. Watson HJ, Bulik CM. Update on the treatment of anorexia nervosa: review of clinical trials, practice guidelines and emerging interventions. Psychol Med. 2013;43(12):2477-500.

17. Zipfel $\mathrm{S}$ et al. Focal psychodynamic therapy, cognitive behaviour therapy, and optimised treatment as usual in outpatients with anorexia nervosa (ANTOP study): randomised controlled trial. Lancet. 2014;383(9912):127-37.

18. Fairburn CG. Cognitive behavior therapy and eating disorders. New York: Guildford Press; 2008.

19. Couturier J, Kimber M, Szatmari P. Efficacy of family-based treatment for adolescents with eating disorders: a systematic review and meta-analysis. Int J Eat Disord. 2013;46(1):3-11.

20. Agras WS et al. Comparison of 2 family therapies for adolescent anorexia nervosa: a randomized parallel trial. JAMA Psychiatr. 2014;71(11):1279-86.

21. Hart LM et al. Unmet need for treatment in the eating disorders: a systematic review of eating disorder specific treatment seeking among community cases. Clin Psychol Rev. 2011;31(5):727-35.

22. Fursland A, Watson HJ. Eating disorders: a hidden phenomenon in outpatient mental health? Int J Eat Disord. 2014;47(4):422-5.

23. Tobin DL et al. I know what you did last summer (and it was not CBT): a factor analytic model of international psychotherapeutic practice in the eating disorders. Int J Eat Disord. 2007;40(8):754-7.

24. Waller G, Stringer H, Meyer C. What cognitive behavioral techniques do therapists report using when delivering cognitive behavioral therapy for the eating disorders? J Consult Clin Psychol. 2012;80(1):171-5. 
25. Wallace LM, von Ranson KM. Perceptions and use of empiricallysupported psychotherapies among eating disorder professionals. Behav Res Ther. 2012;50(3):215-22.

26. von Ranson KM, Wallace LM, Stevenson A. Psychotherapies provided for eating disorders by community clinicians: infrequent use of evidence-based treatment. Psychother Res. 2013;23(3):333-43.

27. Couturier $\mathbf{J}$ et al. Understanding the uptake of family-based treatment for adolescents with anorexia nervosa: therapist perspectives. Int J Eat Disord. 2013;46(2):177-88.

28. Couturier $\mathrm{J}$ et al. Using a knowledge transfer framework to identify factors facilitating implementation of family-based treatment. Int $\mathbf{J}$ Eat Disord. 2014;47(4):410-7.

29. Barlow DH, Levitt JT, Bufka LF. The dissemination of empirically supported treatments: a view to the future. Behav Res Ther. 1999;37:S147-62.

30. Gallo K, Barlow DH. Factors involved in clinician adoption and nonadoption of evidence-based interventions in mental health. Clin Psychol Sci Pract. 2012;93-106.

31. Wilson GT, Zandberg LJ. Cognitive-behavioral guided self-help for eating disorders: effectiveness and scalability. Clin Psychol Rev. 2012;32(4):343-57. A systematic review of the research on guided self-help treatments for eating disorders. The focus is on the use of guided self-help to make eating disorder treatment more available.

32. Simmons AM, Milnes SM, Anderson DA. Factors influencing the utilization of empirically supported treatments for eating disorders. Eat Disord. 2008;16(4):342-54.

33. Mussell MP et al. Utilization of empirically supported psychotherapy treatments for individuals with eating disorders: a survey of psychologists. Int J Eat Disord. 2000;27(2):230-7.

34. Waller $\mathrm{G}$ et al. Attitudes towards psychotherapy manuals among clinicians treating eating disorders. Behav Res Ther. 2013;51(12): $840-4$.

35. Straebler SM, Cooper Z. Training therapists as agents of implementation. Poster presented at: World Congress of Behavioral and Cognitive Psychotherapies. Lima, Peru; 2013.

36. Wallace LM, von Ranson KM. Treatment manuals: use in the treatment of bulimia nervosa. Behav Res Ther. 2011;49(11):815-20.

37. Turner $\mathrm{H}$ et al. Clinicians' concerns about delivering cognitivebehavioural therapy for eating disorders. Behav Res Ther. 2014;57:38-42.

38. Fairburn CG et al. Transdiagnostic cognitive-behavioral therapy for patients with eating disorders : a two-site trial with 60-week followup. Am J Psychiatry. 2009;166(3):311-9.

39. Couturier J, Isserlin L, Lock J. Family-based treatment for adolescents with anorexia nervosa: a dissemination study. Eat Disord. 2010;18(3):199-209.

40. Byrne SM et al. The effectiveness of enhanced cognitive behavioural therapy for eating disorders: an open trial. Behav Res Ther. 2011;49(4):219-26.

41. Knott, $\mathrm{S}$ et al. Cognitive behaviour therapy for bulimia nervosa and eating disorders not otherwise specified: translation from randomized controlled trial to a clinical setting. Behav Cognitive Psychother. 2014; advance online publication (10.1017/ S1352465814000393).

42. Waller $\mathrm{G}$ et al. Cognitive-behavioral therapy for bulimia nervosa and atypical bulimic nervosa: effectiveness in clinical settings. Int $\mathrm{J}$ Eat Disord. 2014;47(1):13-7.

43. Murphy R et al. How do psychological treatments work? Investigating mediators of change. Behav Res Ther. 2009;47(1): $1-5$.

44.• Kazdin AE, Rabbitt SM. Novel models for delivering mental health services and reducing the burdens of mental illness. Clin Psychol Sci. 2013;1:170-19. A wide-ranging discussion about how to meet the challenges of making evidence-based interventions for mental health problems widely available. Particular emphasis is placed on the use of novel methods drawn from fields other than mental health.

45.• Fairburn CG, Patel V. The global dissemination of psychological treatments: a road map for research and practice. Am J Psychiatr. 2014;171(5):495-8. A brief paper providing an outline of potential methods for scaling up available evidence-based treatments within the field of mental health.

46. Mitchell JE et al. A randomized trial comparing the efficacy of cognitive-behavioral therapy for bulimia nervosa delivered via telemedicine versus face-to-face. Behav Res Ther. 2008;46(5): 581-92.

47. Crow SJ et al. The cost effectiveness of cognitive behavioral therapy for bulimia nervosa delivered via telemedicine versus face-toface. Behav Res Ther. 2009;47(6):451-3.

48. Bulik CM et al. CBT4BN versus CBTF2F: comparison of online versus face-to-face treatment for bulimia nervosa. Contemp Clin Trial. 2012;33(5):1056-64.

49. Clark DM. The English improving access to psychological therapies (IAPT) program: history and progress. In: McHugh RK, Barlow DH, editors. Dissemination and implementation of evidence-based psychological interventions. New York: Oxford University Press; 2012. p. 61-77.

50. Ruzek JI, Karlin BE, Zeiss A. Implementation of evidence-based psychological treatments in the Veterans Health Administration. In: McHugh RK, Barlow DH, editors. Dissemination and implementation of evidence-based psychological interventions. New York: Oxford University Press; 2012. p. 78-96.

51. Fairburn CG, Cooper Z. Therapist competence, therapy quality, and therapist training. Behav Res Ther. 2011;49(6-7):373-8.

52. Beidas RS, Kendall PC. Training therapists in evidence-based practice: a critical review of studies from a systems-contextual perspective. Clin Psychol. 2010;17(1):1-30.

53. Herschell AD et al. The role of therapist training in the implementation of psychosocial treatments: a review and critique with recommendations. Clin Psychol Rev. 2010;30(4): 448-66.

54. Cooper Z, Doll HA, Bailey-Straebler S, Kluczniok D, Murphy R, O'Connor ME, et al. The development of an online measure of therapist. Behav Res Ther. 2015;64:43-48.

55. Patel V. The future of psychiatry in low- and middle-income countries. Psychol Med. 2009;39(11):1759.

56. Zandberg LJ, Wilson GT. Train-the-trainer: implementation of cognitive behavioural guided self-help for recurrent binge eating in a naturalistic setting. Eur Eat Disord Rev. 2013;21(3): $230-7$.

57. Fairburn CG, Wilson GT. The dissemination and implementation of psychological treatments: problems and solutions. Int J Eat Disord. 2013;46(5):516-21.

58. van Ginneken N et al. Non-specialist health worker interventions for the care of mental, neurological and substance-abuse disorders in low- and middle-income countries. Cochrane Database of Systematic Reviews. 2013;(11).

59. McHugh RK, Barlow DH. Training in evidence-based psychological interventions. In: McHugh RK, Barlow DH, editors. Dissemination and implementation of evidence-based psychological interventions. New York: Oxford University Press; 2012. p. 43-58.

60. Payne LA et al. Emotional disorders: a unified transdiagnostic protocol. In: Barlow DH, editor. Clinical handbook of psychological disorders: a step-by-step treatment manual. New York: Guildford Press; 2014. p. 237-74.

61. Fairburn CG, Cooper Z. Eating disorders: a transdiagnotic protocol. In: Barlow DH, editor. Clinical handbook of psychological disorders: a step-by-step treatment manual. New York: Guildford Press; 2014. p. 670-702. 
62. Chorpita BF, Daleiden EL. Mapping evidence-based treatments for children and adolescents: application of the distillation and matching model to 615 treatments from 322 randomized trials. J Consult Clin Psychol. 2009;77(3):566-79.

63. Chorpita BF et al. The old solutions are the new problem: how do we better use what we already know about reducing the burden of mental illness? Perspect Psychol Sci. 2011;6(5):493-7.

64. Fairburn CG et al. Enhanced cognitive behaviour therapy for adults with anorexia nervosa: a UK-Italy study. Behav Res Ther. 2013;51(1):R2-8.

65. Sholomskas DE et al. We don't train in vain: a dissemination trial of three strategies of training clinicians in cognitive-behavioral therapy. J Consult Clin Psychol. 2005;73(1):106-15.

66. Dimeff LA et al. Which training method works best? A randomized controlled trial comparing three methods of training clinicians in dialectical behavior therapy skills. Behav Res Ther. 2009;47(11): 921-30.

67. Hart LM et al. Unmet need for treatment in the eating disorders: a systematic review of eating disorder specific treatment seeking among community cases. Clin Psychol Rev. 2011;31(5):727-35.

68. Fairburn CG, Carter JC. Self-help and guided self-help for bingeeating problems. In: Garner D, Garfinkel P, editors. Handbook of treatment for eating disorders. Guildford Press; 1997. p. 494-9.

69. Cuijpers $P$ et al. Is guided self-help as effective as face-to-face psychotherapy for depression and anxiety disorders? A systematic review and meta-analysis of comparative outcome studies. Psychol Med. 2010;40(12):1943-57.

70. Newman MG et al. A review of technology-assisted self-help and minimal contact therapies for anxiety and depression: is human contact necessary for therapeutic efficacy? Clin Psychol Rev. 2011;31(1):89-103.
71. Sysko R, Walsh BT. A critical evaluation of the efficacy of self-help interventions for the treatment of bulimia nervosa and binge-eating disorder. Int J Eat Disord. 2008;41(2):97112.

72. Grilo CM et al. Self-help for binge eating disorder in primary care: a randomized controlled trial with ethnically and racially diverse obese patients. Behav Res Ther. 2013;51(12):855-61.

73. Traviss GD, Heywood-Everett S, Hill AJ. Guided self-help for disordered eating: a randomised control trial. Behav Res Ther. 2011;49(1):25-31.

74. Shingleton RM, Richards LK, Thompson-Brenner H. Using technology within the treatment of eating disorders: a clinical practice review. Psychotherapy. 2013;50(4):576-82.

75. Aardoom JJ et al. Treating eating disorders over the internet: a systematic review and future research directions. Int J Eat Disord. 2013;46(6):539-52.

76. Dölemeyer $\mathrm{R}$ et al. Internet-based interventions for eating disorders in adults: a systematic review. BMC Psychiatr. 2013;13:207.

77. Loucas CE et al. E-therapy in the treatment and prevention of eating disorders: a systematic review and meta-analysis. Behav Res Ther. 2014;63:122-31. A systematic review of the evidence for scalable $e$ therapy interventions for eating disorders using NICE methodology.

78. Bauer S, Moessner M. Harnessing the power of technology for the treatment and prevention of eating disorders. Int J Eat Disord. 2013;46(5):508-15. A review of the role of technology in enhancing the care of those with eating disorders. Helpful recommendations concerning future work are provided.

79. Grilo $\mathrm{CM}$ et al. Treatment of binge eating disorder in racially and ethnically diverse obese patients in primary care: randomized placebo-controlled clinical trial of self-help and medication. Behav Res Ther. 2014;58:1-9. 